\title{
Gemcitabine-Related Acute Coronary Syndrome
}

\author{
Randa Hazam ${ }^{\mathrm{a}, \mathrm{d}}$, Ahmed Taha ${ }^{\mathrm{b}, \mathrm{d}}$, John Kimbugwe ${ }^{\mathrm{a}}$, \\ Roaa Ahmed ${ }^{c}$, Stephen Wright ${ }^{\mathrm{a}}$
}

\begin{abstract}
A strong association of gemcitabine to acute coronary syndrome (ACS) is not currently established in the literature. In this series, we highlight that both patients with gemcitabine-related ACS were more than 50 years old, had underlying coronary artery disease and suffered from metastatic cancer. However, further prospective studies are required to validate the significance of these observations.
\end{abstract}

Keywords: Gemcitabine; Cardiotoxicity; Acute coronary syndrome

\section{Introduction}

Chemotherapy-induced cardiotoxicity ranges from mild events like slight blood pressure (BP) changes, asymptomatic electrocardiographic (EKG) changes and pericarditis, to more serious and potentially lethal events like arrhythmias, myocarditis, cardiomyopathy and acute coronary syndrome (ACS). Gemcitabine is generally a well-tolerated cytotoxic agent with a very low cardiotoxicity profile, with the most common side effect being dose-limiting myelosuppression. Several factors related to gemcitabine (dose and infusion regimen) or the patient (age, electrolyte imbalances, history of cardiac diseases and concurrent use of other cardiotoxic agents) can influence the incidence of gemcitabine-related ACS [1]. Gemcitabine-related ACS is extremely rare, and only a few case reports are present in the literature. Despite being uncommon, it should be taken into consideration by physicians as prompt intervention could prevent a potentially lethal outcome.

Manuscript submitted January 8, 2020, accepted January 17, 2020

aDepartment of Internal Medicine, Texas Tech University Health Sciences Center, Amarillo, TX, USA

${ }^{b}$ Department of Internal Medicine, Deaconess Hospital, Evansville, IN, USA 'School of Medicine, Ahfad University for Women, Omdurman, Sudan

${ }^{\mathrm{d} C o r r e s p o n d i n g ~ A u t h o r: ~ R a n d a ~ H a z a m, ~ D e p a r t m e n t ~ o f ~ I n t e r n a l ~ M e d i c i n e, ~}$ Texas Tech University Health Sciences Center, 1400 S Coulter St, Suite 2700, Amarillo, TX 79106, USA. Email: randa.hazam@ttuhsc.edu; Ahmed Taha, Department of Internal Medicine, Deaconess Hospital, 600 Mary St., Evansville, IN 47747, USA. Email: aaataha1@gmail.com

doi: https://doi.org/10.14740/jmc3417

\section{Case Reports}

\section{Case 1}

A 68-year-old man presented with constant non-radiating chest tightness that was relieved with nitroglycerin. No cough, shortness of breath, or hemoptysis were noted. He had history of diabetes mellitus, hypertension, chronic kidney disease, peripheral vascular disease and stage IV lung cancer (cT2, N0, M1) with metastases to the left jugular lymph nodes. On examination, temperature was $37.5^{\circ} \mathrm{C}$, oxygen saturation was $94 \%$ on room air, respiratory rate (RR) was $19 / \mathrm{min}$, heart rate (HR) was 80 beats per minute (bpm) and BP was $114 / 52 \mathrm{~mm}$ Hg. Chest, abdominal and cardiovascular examinations were unremarkable. No lower extremities edema was noted. Five days prior to presentation, patient received gemcitabine 2,280 $\mathrm{mg}$ and vinorelbine $57 \mathrm{mg}$. EKG showed ST-segment depression in leads V3-V5. Serum troponin I was $21.6 \mathrm{ng} / \mathrm{mL}$, total white blood cell count (TWBC) was $5,600 / \mu \mathrm{L}$, hemoglobin was $9.2 \mathrm{~g} / \mathrm{dL}$, platelet count was $161,000 / \mu \mathrm{L}$, serum potassium $(\mathrm{K})$ was $4 \mathrm{mEq} / \mathrm{L}$, serum magnesium $(\mathrm{Mg})$ was $2.3 \mathrm{mg} /$ $\mathrm{dL}$ and serum creatinine was $2.2 \mathrm{mg} / \mathrm{dL}$ (estimated glomerular filtration rate (eGFR) was $40 \mathrm{~mL} / \mathrm{min}$ (baseline creatinine 1.3 $\mathrm{mg} / \mathrm{dL})$ ). Chest X-ray was normal. The patient was admitted to the intensive care unit (ICU) with a working diagnosis of non-ST-segment elevation myocardial infarction (NSTEMI) and managed with aspirin, clopidogrel, metoprolol, simvastatin and heparin bolus followed by infusion (due to reduced GFR). Chest pain resolved with medical treatment and troponin I trended down to $4.98 \mathrm{ng} / \mathrm{mL}$ over 3 days. No invasive revascularization (percutaneous coronary intervention (PCI) or coronary artery bypass grafting (CABG)) was done and gemcitabine and vinorelbine were held for 3 weeks.

\section{Follow-up}

For a 1-month period, the patient remained chest pain-free for 1 month, thereafter, a second cycle of gemcitabine was administered, followed by a third cycle 1 week later. Four days after receiving the third cycle of gemcitabine, he was found somnolent and confused, in atrial fibrillation (AF) with rapid ventricular response, and HR of $150 \mathrm{bpm}$. He was found to have significant neutropenia, septic shock, severe acute kidney injury and significant lactic acidosis. He then had cardiac arrest with pulseless electrical activity and passed away. 


\section{Case 2}

An 84-year-old man had a remote history of coronary artery disease (CAD) and four-vessel coronary bypass surgery (20 years ago) and a recent diagnosis of stage IIA, $2.5-\mathrm{cm}$ pancreatic adenocarcinoma (T3, N0, M0). He was planned for chemotherapy with three cycles of gemcitabine weekly $(1,950$ $\mathrm{mg} / \mathrm{m}^{2} /$ cycle) followed by stereotactic radiation therapy as neoadjuvant therapy. Two days after the second cycle of gemcitabine, the patient presented with generalized weakness, cold extremities, diaphoresis and shortness of breath. No chest pain or palpitations was noted. Pulse oximeter revealed oxygen saturation $84 \%$ on room air, BP $84 / 56$, jugular venous distension and bibasilar lung crackles. Laboratory tests revealed serum creatinine kinase-MB $54 \mathrm{ng} / \mathrm{mL}$, troponin I $32 \mathrm{ng} / \mathrm{mL}$, serum $\mathrm{K} 4.5 \mathrm{mEq} / \mathrm{L}$, serum $\mathrm{Mg} 1.8 \mathrm{mg} / \mathrm{dL}$, serum creatinine 1.75 (eGFR $37 \mathrm{~mL} / \mathrm{min}$ ) (baseline creatinine $1.3 \mathrm{mg} / \mathrm{dL}$ ) and TWBC $16,100 / \mu \mathrm{L}$ (96.9\% neutrophils). EKG showed new Twave inversion in leads V3-6. Chest X-ray showed pulmonary edema and transthoracic echocardiogram (TTE) showed ejection fraction of $35 \%$ with global hypokinesis. The patient was admitted to the ICU with the diagnosis of NSTEMI and cardiogenic shock. Patient required vasopressor support with norepinephrine and was also treated with aspirin, plavix, therapeutic low-molecular-weight heparin. No invasive revascularization (PCI or $\mathrm{CABG}$ ) was done.

\section{Follow-up}

Patient's clinical condition improved, cardiac enzymes trended down and he was discharged in a stable condition. The patient then lost follow-up with oncology department but returned 1 year later with peritoneal carcinomatosis, referred to hospice where he passed away couple of months later.

\section{Discussion}

Gemcitabine is a highly liposoluble molecule, and its clearance yields a primary deamination metabolite, difluorodeoxyuridine (dFdU). Gemcitabine's pharmacokinetics and distribution volumes depend on infusion modality and are influenced by patient's factors that include age, gender, infusion length and body surface area [2]. There is a paucity of data regarding gemcitabine cardiotoxicity and a review of phase II clinical trials showed a low cardiotoxic profile and extremely rare cardiac events after gemcitabine infusion: ventricular tachyarrhythmias in $0-1.4 \%$, reduction of left ventricular ejection fraction in $0.2-0.9 \%$ and exudative pericarditis in $0.2 \%$ [3]. Several chemotherapy agents were initially deemed safe from the cardiac perspective during phase I and phase II trials but incited a trigger for further pharmacovigilance surveys upon their widespread use. Our case series is introducing a probe to further investigate gemcitabine cardiotoxicity, particularly, ACS.

ACS is an extremely rare manifestation of gemcitabinerelated cardiotoxicity with only four cases previously reported in the literature to date; hereby, we are reporting the fifth and sixth cases in Table 1 [4-7]. Most patients were older than 50 years and had metastatic cancer. Save for one case, all ACS incidences occurred after at least the second gemcitabine infusion cycle, and the time interval between the infusion to the onset of symptoms varied between $30 \mathrm{~min}$ to 5 days. Interestingly, $50 \%$ of the patients had pre-existing CAD.

Due to the scarcity of data linking gemcitabine to ACS, no cause-effect relationship was established. However, besides the absence of other potential etiologies, a strong temporal and clinical association of gemcitabine to the evolution of ACS was observed in all reported cases, including both of our present cases. Additionally, there is no current data to interpret the great variation in time-interval (infusion-to-onset of symptoms) of $30 \mathrm{~min}$ to 5 days. There is no evidence to explain the high incidence of ACS among those with pre-existing CAD, either. Therefore, careful clinical and cardiac monitoring during and after gemcitabine infusions seems warranted, especially if the patient has an underlying CAD. Further clinical studies are required to validate these observations though.

Though the mechanisms of gemcitabine-related myocardial ischemia are not well understood, some authors have speculated direct endothelial injury resulting in acute coronary thrombosis as a possible mechanism. The evidence for prothrombotic and procoagulant effects of gemcitabine comes from other macrovascular events such as strokes, visceral infractions and vasculitides that were reported in association with gemcitabine infusion [7]. Kalapura et al described higher incidence of thrombosis and vascular events in patients treated with gemcitabine. There is also some conjecture about coronary vasospasm as being another possible pathogenetic mechanism. It was proposed by Ozturk et al as a cause of chest pain and a new-onset left bundle branch block during gemcitabine infusion [4]. In that particular case, vasospasm, chest pain and EKG changes had resolved within $10 \mathrm{~min}$ of antianginal therapy.

In this report, we investigated the extremely rare, yet potentially fatal, gemcitabine-related ACS. Most patients who develop gemcitabine-related ACS were older than 50 years, had metastatic malignancy and had underlying CAD.

\section{Conclusions}

This case series introduces a probe to further investigate the rare gemcitabine-induced ACS. The mechanisms of gemcitabine-induced myocardial ischemia are not well understood; however, direct endothelial injury and coronary vasospasm are the widely adopted theories. Therefore, careful clinical and cardiac monitoring during and after gemcitabine infusions seems warranted. Most patients who developed gemcitabinerelated ACS were older than 50 years, had metastatic cancer and had underlying CAD. Further clinical studies are required to validate the clinical significance of these observations.

\section{Acknowledgments}

None to declare. 


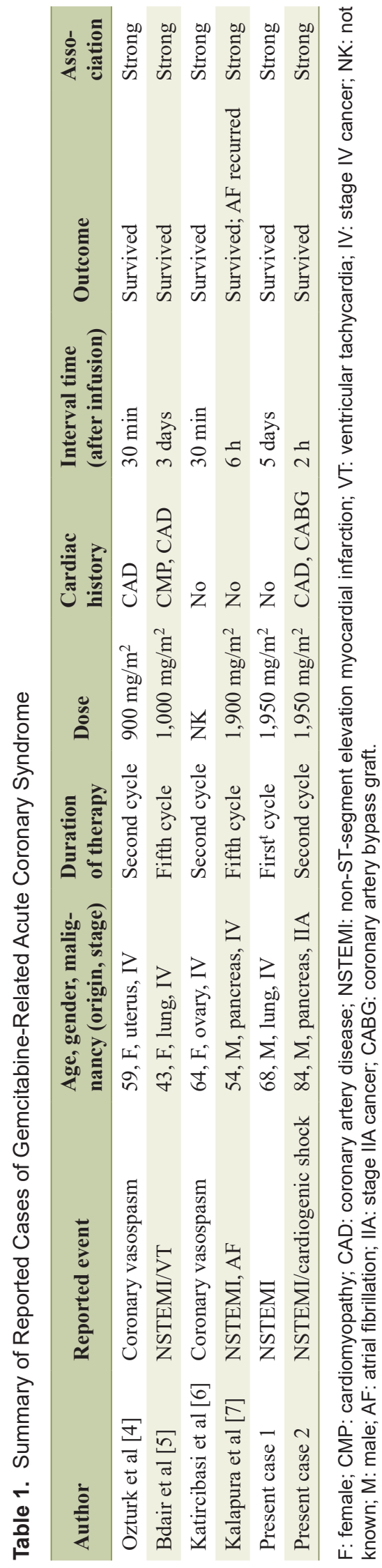

\section{Financial Disclosure}

None to declare.

\section{Conflict of Interest}

The authors have no conflict of interest to disclose.

\section{Informed Consent}

Verbal informed consent was obtained from the patient(s) for their anonymized information to be published in this article.

\section{Author Contributions}

$\mathrm{RH}, \mathrm{AT}$ and SW contributed to the acquisition and interpretation of the data; drafting and critically revising the manuscript; approving the final publishable version of the manuscript; and agreeing to be accountable for all aspects of the work. JK contributed to the acquisition of the data; drafting the manuscript; approving the publishable version of the manuscript; and agreeing to be accountable for all aspects of the work. RA contributed to drafting the manuscript; approving the final publishable version of the manuscript; and agreeing to be accountable for all aspects of the work.

\section{Data Availability}

The authors declare that data supporting the findings of this manuscript are available within the article. Also, any inquiries regarding supporting data availability of this article should be directed to the corresponding authors.

\section{Abbreviations}

ACS: acute coronary syndrome; CAD: coronary artery disease; BP: blood pressure; EKG: electrocardiogram; RR: respiratory rate; HR: heart rate; bpm: beats per minute; TWBC: total white blood cell; eGFR: estimated glomerular filtration rate; ICU: intensive care unit; NSTEMI: non-ST-segment elevation myocardial infarction; PCI: percutaneous coronary intervention; $\mathrm{CABG}$ : coronary artery bypass graft; $\mathrm{AF}$ : atrial fibrillation; TTE: transthoracic echocardiogram; EF: ejection fraction; dFdU: difluorodeoxyuridine; VT: ventricular tachycardia; CMP: cardiomyopathy

\section{References}

1. Yeh ET, Tong AT, Lenihan DJ, Yusuf SW, Swafford J, Champion C, Durand JB, et al. Cardiovascular compli- 
cations of cancer therapy: diagnosis, pathogenesis, and management. Circulation. 2004;109(25):3122-3131.

2. Dumontet C, Morschhauser F, Solal-Celigny P, Bouafia F, Bourgeois E, Thieblemont C, Leleu X, et al. Gemcitabine as a single agent in the treatment of relapsed or refractory low-grade non-Hodgkin's lymphoma. Br J Haematol. 2001;113(3):772-778.

3. Tonato M, Mosconi AM, Martin C. Safety profile of gemcitabine. Anticancer Drugs. 1995;6(Suppl 6):27-32.

4. Ozturk B, Tacoy G, Coskun U, Yaman E, Sahin G, Buyukberber S, Yildiz R, et al. Gemcitabine-induced acute coronary syndrome: a case report. Med Princ Pract. 2009;18(1):76-80.

5. Bdair FM, Graham SP, Smith PF, Javle MM. Gemcitabine and acute myocardial infarction - a case report. Angiology. 2006;57(3):367-371.

6. Katircibasi MT, Eken A. Gemcitabine-induced coronary vasospasm: A case report. Turk Kardiyol Dern Ars. 2017;45(2):172-175.

7. Kalapura T, Krishnamurthy M, Reddy CV. Acute myocardial infarction following gemcitabine therapy - a case report. Angiology. 1999;50(12):1021-1025. 